\title{
Motor Current Demodulation Analysis applied with Neural Networks and Genetic Algorithms for Rotor Bar Faults Diagnosis
}

\author{
Z.M. Taïb ${ }^{1}$, M. Hasni ${ }^{2}$, O Touhami ${ }^{1}$, R. Ibtiouen $^{l}$ \\ ${ }^{I}$ LRE-ENPolytechnique d'Alger, 10 av. Pasteur El Harrach, BP.182, 16200 Alger \\ e-mail: omar.touhami@enp.edu.dz \\ ${ }^{2}$ LSEI - USTHB- Université des sciences et de la technologie H. Boumediene, BP.32 El-Alial6111 Algiers \\ e-mail: hasnimourad2001@yahoo.fr.,mhasni@usthb.dz
}

\begin{abstract}
Our Work come in forward of several investigations in motor diagnosis field combining the use of Motor current demodulation analysis (MCDA) and the multilayer feed-forward artificial neural networks (ANNs) optimized by genetic algorithms (GAs). ANNs are used effectively to recognize Broken Rotor bar Faults severity. The novelty in this work is that proposed methodology is based on the extraction of fault components from the spectrum of both current amplitude and phase modulation (AM and PM), AM and PM signals are extracted by signal demodulation techniques from the measured stator current ; this methodology have provided more accurate results on faulty tested motors at different loads.
\end{abstract}

\section{Key words}

Induction machine, Faults diagnosis, ANNS, signal modulation.

\section{Introduction}

Nowadays, The Induction motors (IM) become the work horses for the industry due to their strength, low maintenance cost and easy operational supply; However, in practical applications, the (IM) are subject to inevitable stresses that may cause defects on several (IM) parts threatening the normal production operation. As a result, manufacturing companies are working hard to find incipient fault detection techniques in order to install earlier Maintenance plans based primarily on status monitoring of the (IM), [1-3]. Investigations conducted by the Electric Power Research Institute (EPRI) [4] and IEEEIAS [5], have shown that most motor failures are mainly caused by two fault types: Broken Rotor bars Fault and Stator winding Inter turns. This paper discusses the fault diagnosis of induction machine using Motor current demodulation analysis (MCDA) techniques where the monitoring process is mainly conducted by stator current signal analysis that leads to extract significant fault components. To avoid too much instrumentation, the sensors used are those conventional, necessary to control the electrical machines, namely the current sensors. A supervised artificial neural network (ANN) has been adopted for fault classification part because it is the more efficient intelligent technique used in pattern recognizing field, [6-11]. The networks are trained and tested using a data base constructed by the extraction of fault components. Genetic Algorithm (GA) Was adopted to find the optimum architecture and to develop novel criteria that quantify the ANN's performance as well as its complexity [9]. This approach is implemented in software and tested. Results have shown that the approach offers many advantages by comparison to similar approaches based on other MCSA techniques and proves that the proposed neural network approach combining with MCD analysis is promising for detecting machine faults

\section{Signal Processing And Features Extraction}

\section{A. Moteur Current Demodulation Analysis}

Many researchers [12-17] dealing with MCSA techniques for motor diagnosis have been established in basis of using Fast Fourier Transformation (FFT) because it requires a simple and inexpensive calculation tools, this spectrum technique is mainly used to detect fault signature components; however, this technique have shown no accurate results with non-loaded motors or with frequency disturbance caused by motor vibrations that affects greatly fault components in current spectrum. This is way many research [18-20] have been conducted to overcome this weakness by Motor Current Demodulation Analysis technique. The basic idea of MCDA technique is to execute during analysis a signal demodulation; this means 
that to extract some courant signal parts that are related directly to the faults and make analysis only on these parts. Authors in references [18-20] have stated that with rotor broken bars fault both current amplitude modulation and phase modulation (AM and PM) should coexist; this mixture can be explained by a complex modulation which changes in the same time the amplitude and the phase as follow:

$$
\begin{gathered}
i_{a}=I_{A P}\left(1+I_{A M}(t)\right) \\
i_{A M}=I_{m}\left(\omega t-\varphi_{1}\right)\left(1+I_{A}(t)\right) \\
i_{A P}=I_{m}\left(\omega t+I_{P}(t)\right)
\end{gathered}
$$

$I_{A P}$ and $I_{A M}$ are respectively phase and current modulated signals. Both of the modulating signals in amplitude $I_{A}(t)$ and in phase $I_{p}(t)$ are function of pole slip frequency, Which is in the same time the fault frequency:

$f_{S P}=p f_{\text {slip }}=p g f_{\text {sync }}=2 g f_{s}=2 f_{s}-f_{r} p$

With MCDA techniques we should be able to extract current modulating signals in amplitude and in phase separately, and for that we have chosen Hilbert Transformation. Mathematically, Hilbert Transformation of a real signal as a current signal is defined as follow:

$$
y(t)=H T\{x(t)\}=\frac{1}{\pi t} \int_{-\infty}^{+\infty} \frac{x(\tau)}{t-\tau} d \tau
$$

If we associate both real signal and its Hilbert transformation $\mathrm{x}(t)$ and $y(t)$, we generate what we call analytic signal:

$$
\dot{x}(t)=x(t)+j y(t)=a(t) e^{j \theta(t)}
$$

with:

$$
a(t)=\sqrt{\left(x^{2}(t)+y^{2}(t)\right)}
$$

$a(t)$ is the instantaneous amplitude of the signal $\mathrm{x}(\mathrm{t})$ which defines the amplitude modulation of the signal $x(t)$, and:

$$
\theta(t)=\arctan \left[\frac{x(t)}{y(t)}\right]
$$

$\theta(t)$ is the instantaneous phase of the signal $x(t)$ which defines the phase modulation of the signal $x(t)$, and then, if we carry out a spectrum signal processing on these two signals we can extract easily direct fault frequencies and Modules.

\section{B. Fault components and datbase}

In order to validate this previous theoretical analysis, we have conducted experimental tests on three Induction motors :

1- 1BBMF : motor with two broken bars

2- 2BBMF : motor with one broken bar

3- MWF: Safe Motor.

The tests were conducted to extract stator motor signals thanks to a sensitive Hall effect sensors and an acquisition chart IOTEQ/DAQ series 1005 with a maximum sampling frequency of $200 \mathrm{kHz}$; the tests done on each motor type eighteen times for three different loads which have conducted to have a valuable test set up. Applying demodulation analysis as previously explained leaded to the following results. If we focus on the fig .1 we can notice clearly on both AM spectrum and especially in PM spectrum the fault frequency appeared in the low frequency band, with comparison to the spectrum of the real current signal the identification is really hard unless we have conduct sleep computation. In fig 2 and with the same motor none loaded the fault band is clear only in PM spectrum and less in AM spectrum ; the real current signal spectrum is totally empty of fault components .

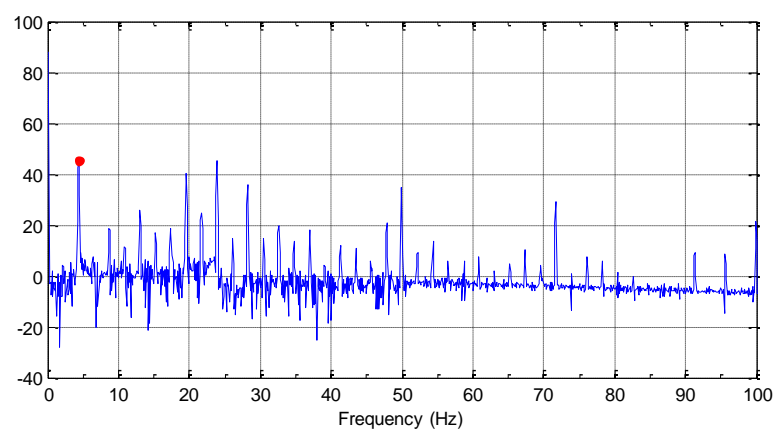

(a)

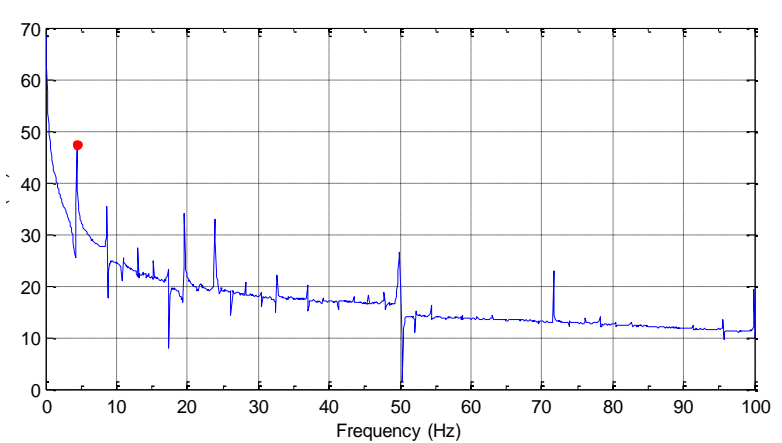

(b)

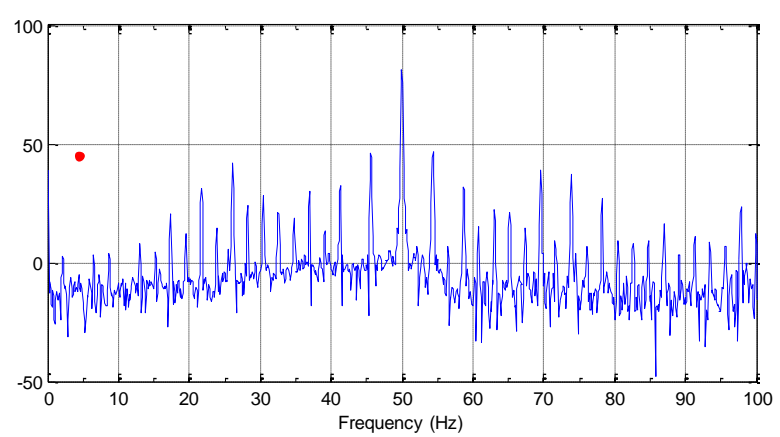

(c)

Fig. 1. Experiments done on 2BBMF motor with full load. (a) Amplitude Demodulation signal spectrum.

(b) phase Demodulation signal spectrum.

(c) Real current signal spectrum.

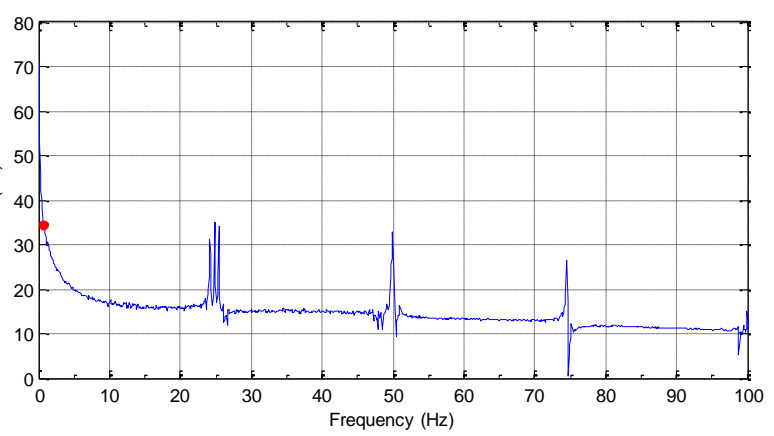

(a) 


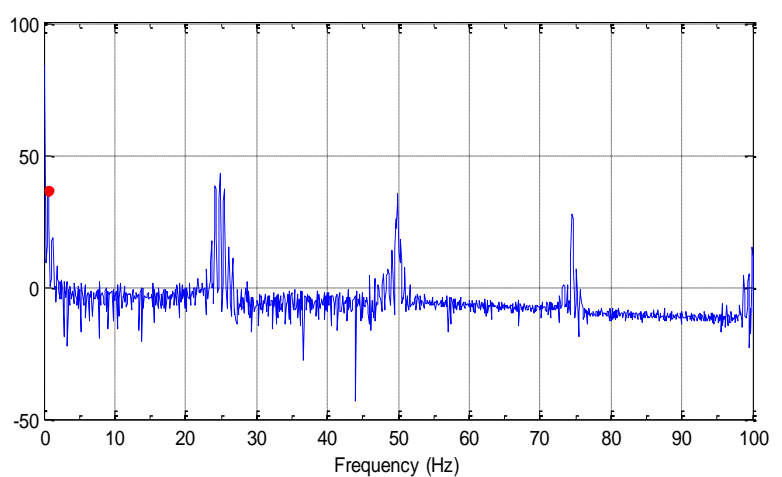

(b)

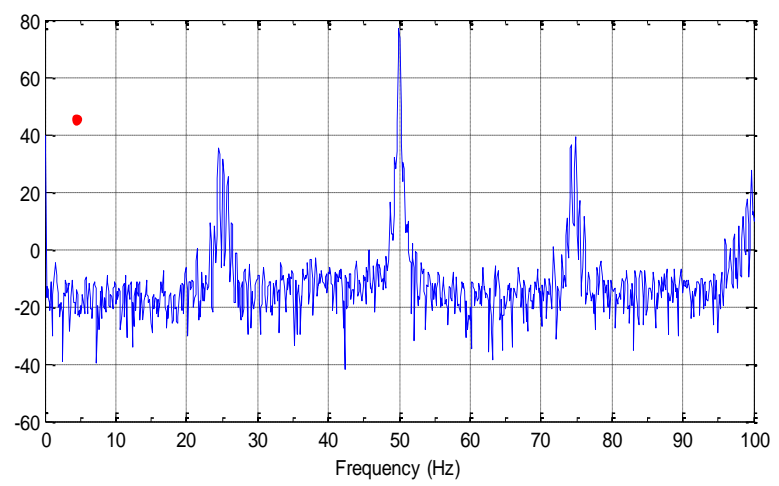

(c)

Fig. 2. Experiments done on $2 B B M F$ motor with no load. (a)Amplitude Demodulation signal spectrum. (b)phase Demodulation signal spectrum.

(c) Real current signal spectrum.

In the same way, the fig 3 and with the safe motor the fault frequency band is also clear in both AM and PM spectrum in contrast in current signal spectrum.

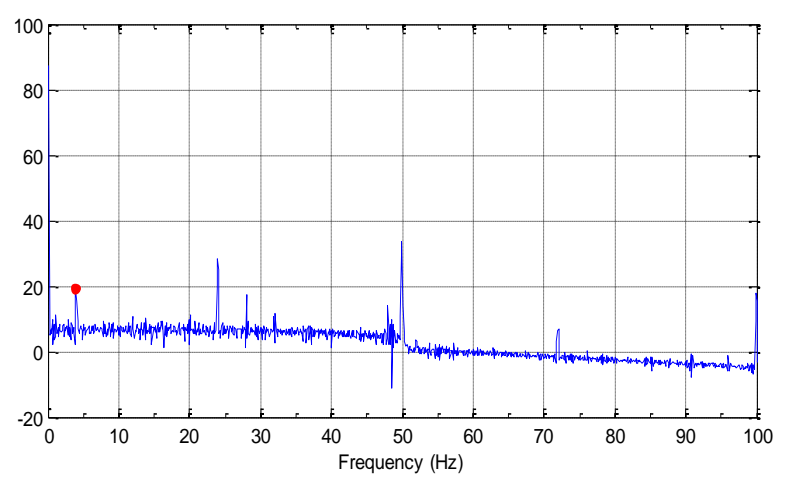

(a)

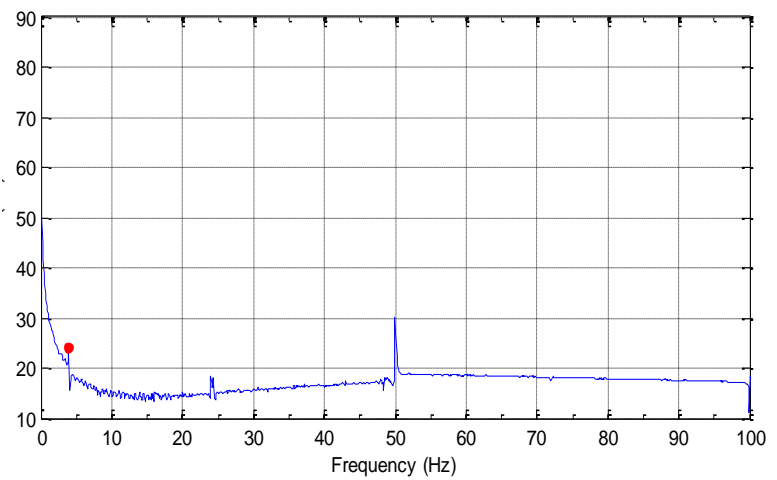

(b)

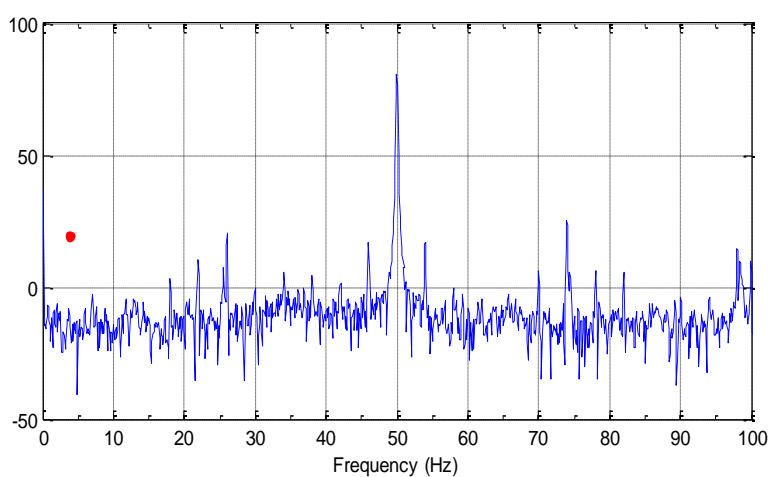

(c)

Fig. 3. Experiments done on MWF motor with full load.

(a) Amplitude Demodulation signal spectrum.

(b) phase Demodulation signal spectrum.

(c) Real current signal spectrum.

In order to develop a fault component database that will be used for ANN's architecture enhancement, we have extracted using the previous MCDA technique- from all current signals set up the following information:

- $\quad$ The slip $« \mathrm{~g} »$.

- Frequency and Amplitude of the fault band characterized by the following formula $f_{s c}=2 g f_{s}$, in both AM and PM spectrum.

This provides six components for each current set up. The extraction of the bands defined previously is done by a MATLAB program which has developed; an example is shown in Fig.4.

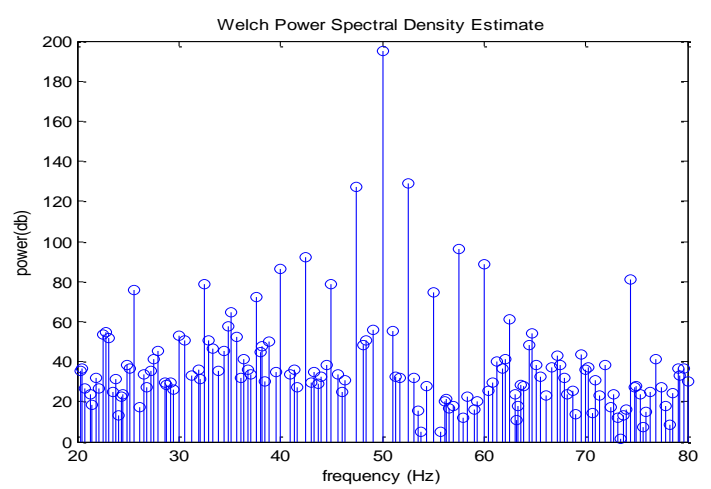

Fig. 4. Extraction of all the peaks of the signal spectrum

The data are then standardized in the interval [-1 1], this comes from the choice taken for the activation function of the Feed-Forward NN layers, for that purpose we used the following function:

$y=\left(y_{\max }-y_{\text {min }}\right) \cdot\left(x-x_{\text {min }}\right) /\left(x_{\max }-x_{\min }\right)+y_{\text {min }}$

$y_{\max }=1 ; x_{\min }:$ the minimal value of vector $x$

$y_{\min }=-1 ; x_{\max }:$ the maximum value of vector $x$

During the training phase of the ANNs we allotted to each input vector a target as a code in 2 bits relatively to each motor type. The data base is then divided in three sets: $70 \%$ training, $15 \%$ validation , $15 \%$ Testing 


\section{Artificial Neural Networks (ANNs)}

The researches have been elaborated in the construction of the ANNs to solve various problems such as the pattern recognition, the forecast, the optimization, the associative memories or the control [8, 9 and 11]. The artificial neural networks are highly connected network of elementary processors running in parallel. Each elementary processor computes a single output based on information it receives. Two main elements constitute an artificial neural network, first on the neuron model used to build the network and then the network architecture. Each artificial neuron is an elementary processor that receives a number of neural inputs upstream. each of these inputs has an associated weight representing the strength of connections between neurons corresponding. This puts forward two specific characteristics of each neuron: a "potential" equal to the weights sum of the inputs and an "activation function" which gives the output of the neuron according to its "potential" [20-22]. The supervised training is used to determine the synaptic weights from labeled examples associated by an expert with network targets. A general architecture of Feed-Forward neural networks as well as the component definitions of the ANNs is shown in Fig.1

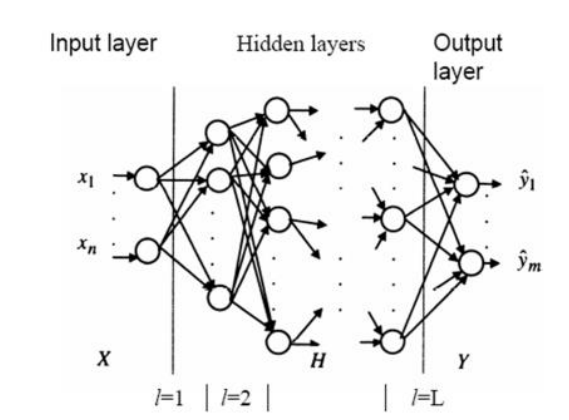

Input Layer: Size depends on problem dimensionality.

Hidden Layer: A design parameter; must decide on number of layers and size for each layer. Creates a non linear generalized decision boundary.

Output Layer: Size depends on number of classification categories.

Bias: Further generalizes the decision boundary.

Net Activation: Weighted sum of the input values at respective hidden nodes.

Activation function: Decides how to categorize the input to a node into a possible node output incorporating the most suitable nonlinearity.

Network Learning: Training an untrained network. Several training methods are available.

Fig 5. A general architecture of Feed-Forward $\mathrm{NN}$ and the component definitions of the ANN, [6,17].

\section{Genetic Algorithms (GAs)}

The genetic algorithms were developed to find optimal solutions in big spaces of research and were used in several sectors as artificial intelligence methods optimization. Several methods of GA were presented to solve major problems and to find solutions of a better quality, and they were applied successfully to solve many combinative problems. Because of the complexity of the problem nature, the genetic algorithm is used to search the solution space of the best architecture defined by a set of criteria. The basic idea of the GAs is that the optimal solution will be founded in solution space areas containing good solutions and these areas can be identified by robust sampling. Practically, this means that the solution space is divided into subsets that are evaluated to find the best solution. The sampling process is performed repeatedly, until finding the best solutions for each subset and then the optimal solution is found.

The Described methodology was implemented in MATLAB environment. The objective function is prepared by combining each of the four criteria described in ref [9]. It is the function that returns a value which must be reduced by the GA and it characterizes the fitness of each FFNN:

$$
\begin{gathered}
\text { ObjVal }=F F A C \times \operatorname{Solspc} \times\left(E_{\text {generalization }}+E_{\text {training }}\right) \\
F F A C=e^{f(x)}
\end{gathered}
$$

Solspc: Solution space consistency criterion

$$
\begin{aligned}
& E_{\text {generalization }}: \text { Generalization error. } \\
& E_{\text {training }} \text { : Training error }
\end{aligned}
$$

\section{Results And Discussions}

The predefined GA program is executed and the following result has been returned back by MATLAB Software:
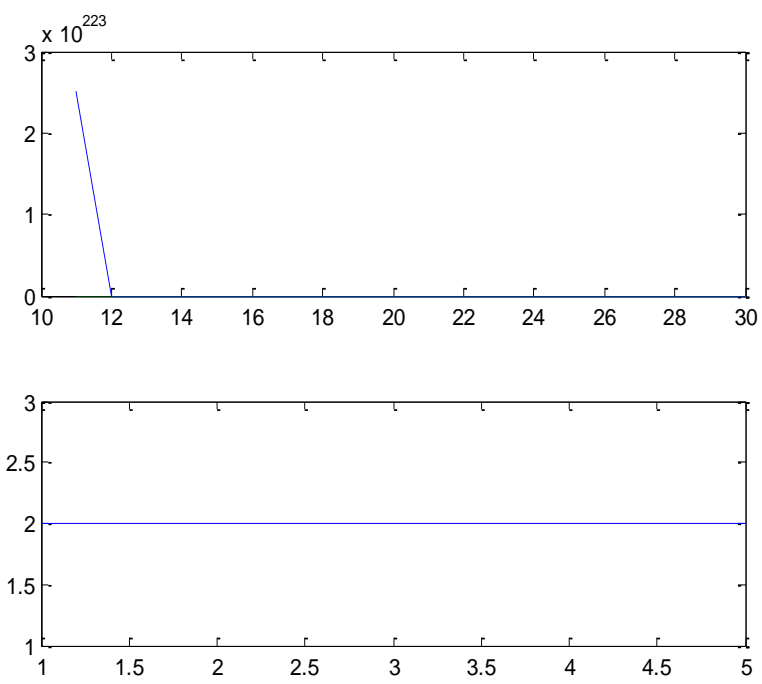

Fig. 6. GA software execution performance and error
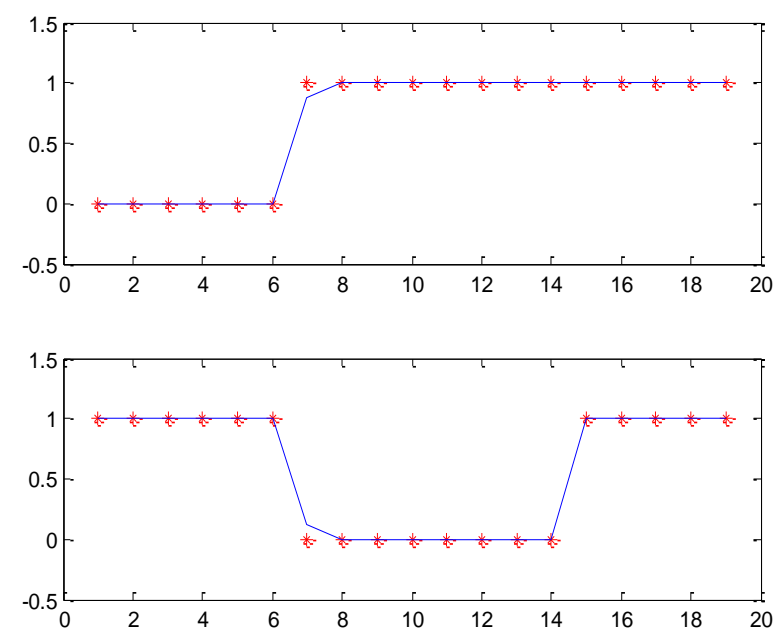

Fig. 7. ANN output target simulation results for with testing set. 
As shown in table I. the resulted architecture is not really complex with only ten nodes ; the testing test is then applied on this architecture the result is that all decision are correct and the rate of success found is $100 \%$.

Table I. ANN architecture result and its performance

\begin{tabular}{|c|c|c|c|}
\hline Architecture & $\begin{array}{c}\text { Objectif } \\
\text { Value }\end{array}$ & Error & $\begin{array}{c}\text { Rate } \\
\text { of success }\end{array}$ \\
\hline $6 \mathrm{X} 2 \mathrm{X} 2$ & 123.8034 & $7.4483 \mathrm{e}-004$ & $100 \%$ \\
\hline
\end{tabular}

\section{Conclusion}

This Work have come in forward to enhance the use of ANN and GA for motor fault diagnosis combining it with a novel MCSA technique ; the Motor current demodulation analysis (MCDA) have proven accurate results validated by experimental set up in LRE laboratory-Ecole Nationale Polytechnique.

The results show that the GA approach performs better than a human expert, and at the same time offers many advantages by comparison to similar approaches found in literature

The results showed the efficiency of GAs for the optimization of the ANNs given the high success rate. We also report the simplicity of the ANNs architecture characterized by the small number of neurons in both hidden layers.

We can consider to use and apply the method on other rotor speed frequencies using the speed inverter.

\section{Appendix}

The name plate data of the squirrel cage induction machine are: Rated Power $\mathrm{P}=4 \mathrm{~kW}$; Rated Voltage $\mathrm{V}_{\mathrm{s}}=220 / 380 \mathrm{~V}(\Delta / \mathrm{Y})$; Rated Current Is $=15.2 / 8.8 \mathrm{~A}$; Rated Speed $\mathrm{Nn}=1435$ (rpm); Pole-pair number $\mathrm{p}=2$; Number of phases $\mathrm{m}=3$; Supply Frequency $\mathrm{f}=50 \mathrm{~Hz} ; \cos \Phi=0.83$; Rotor inertia $\mathrm{J}=0.025 \mathrm{~kg} \cdot \mathrm{m}^{2}$

\section{References}

[1] Motor Reliability Working Group of IEEE. Report of large motor reliability survey of industrial and commercial installations. Part. I and II, IEEE Trans. On industry Applications, vol.IA-21, July/August 1985, pp.853-872.

[2] A.H. Bonnett, G.C. Soukup, Analysis of rotor failures in squirrel cage induction motors, IEEE Transactions on Industry Applications 24 (6) (1988), pp. 1124-1130.

[3] O. V. Thorsen, M. Dalva, "A survey of Fault in induction motors in offshore oil industry, petrochemical industry, gas terminal and oil refineries", IEEE Trans. On Industry applications, vol. 31, n5, 1995, pp. 1-9.

[4] P. F. Albrecht, J. C. Appiarius, and D. K. Sharma, "Assessment of the reliability of motors in utility applications-Updated, " IEEE Transactions on Energy Conversion, vol. 1, pp. 39-46, 1986.

[5]I. C. Report, "Report of large motor reliability survey of industrial and commercial installation, Part I and Part II, “ IEEE Transactions on Industry Applications, vol. 21, 1985, pp. 853-872.

[6] T. Aroui, Y. Koubaa, A. Toumi, " Application of Feed-forward Neural Network for Induction Machine Rotor Faults Diagnostics using Stator Current ", Journal of Electrical Systems (JES), Vol.3, $\mathrm{n}^{\circ} 4,2007$, pp. 213-226.
[7] B. Zhang, J. Yan, W. Zhang, "Application of Multi-layer Feedforward Neural Network in Fault Diagnosis Based on FBP Algorithm", Software Engineering, Artificial Intelligence, Networking, and Parallel/Distributed Computing, SNPD '08. Ninth ACIS International Conference, 2008, pp. 117 - 120.

[8] Fiorenzo Filippetti, Giovanni Franceschini, and Carla Tassoni, "Neural Networks Aided On-Line Diagnostics of Induction Motor Rotor Faults “, IEEE Trans. On Ind. Appl., vol. 31 (4), july/august 1995 , pp. $892-899$

[9] P.G. Benardos, G.-C. Vosniakos "Optimizing feed-forward ANN architecture", science direct, Engineering application of artificial intelligence, Volume 20 (3), Pergamon Press, Inc. Tarrytown, NY, USA, April 2007.

[10] Arabac1 H., "The Detection of Broken Rotor Bars in Squirrel Cage Induction Motors Based on Neural Network Approach", M.S. Selçuk University Graduate School of Natural and Applied Sciences, Konya, 2005.

[11] Vapnik, V. "An overview of statistical learning theory". IEEE Trans. On Neural Networks, vol.10, n5, pp. 988-999, sept.1999.

[12] Touhami, O. and Fadel, M. "Detection of broken rotor bars and stator faults in squirrel cage induction machine". IEEE-IEMDC, Turkey, vol.1, 3-5 May 2007, pp.821-825.

[13] A.M. da Silva, R. J. Povinelli, and N.A.O. Damerdash. "Induction Machine Broken Bar and Stator Short-Circuit Fault Diagnostics Based on Three-phase stator Current Envelopes. IEEE Trans. on Industrial Electronics, vol.55, pp.1310-1318, 2008.

[14] Dulce F. Pires, V. Fern Pires J.F. Martins, A.J. Pires, "Rotor cage fault diagnosis in three-phase induction motors based on a current and virtual flux approach", Energy conversion and management , vol. 50, nº 4, Elsevier, Kidlington UK, pp. 1026-1032, 2009.

[15] Sri Kolla, Logan Varatharasa, Identifying three-phase induction motor faults using artificial neural networks, ISA Transactions, Elsevier Science Ltd, 2000

[16] Hasni, M. ; Hamdani, S. ; Taibi, Z.M. ; Touhami, O. ; Ibtiouen, R. ;Rezzoug, A. "Faults diagnosis of induction machine by using feedforward neural networks and genetic algorithms" 9th Asian Control Conference (ASCC), 2013, pp. $1-6$.

[17] Hasni, M. ; Hamdani, S. ; Taibi, Z.M. ; Touhami, O. ; Ibtiouen, R. ;Rezzoug, A. "Optimization of the feedforward neural network for rotor cage fault diagnosis in three-phase induction motors" IEEE International Electric Machines \& Drives Conference (IEMDC), 2011, pp. 194 - 199

[18] Jaksch. I., Fuchs. P: "Rotor faults detection in induction motors by Motor Current Demodulation Analysis", In Proceedings of SDEMPED 2007, Cracow, Poland, September 2007, pp.247-252.

[19] Jaksch, I, Fuchs, P.: Demodulation Analysis for Exact Rotor Faults Detection under Changing Parameters, SDEMPED 2009, Cargese, France, September 2009, TF0018, IEEE Cat. Number: CFP09SDECDR.

[[20] Ali Zilouchian and Mohammad Jamshidi, "Intelligent control systems using soft computing methodologies", CRC Press LLC, United States of America, 2001.

[21] Hung T. Nguyen, Nadipuram R. Prasad, Carol L. Walker and Elbert A. Walker,"A First Course in Fuzzy and Neural Control" Chapman \& Hall/CRC, 2003.

[22] Abhinav Saxena et Ashraf Saad, "Evolving an artificial neural network classifier for condition monitoring of rotating mechanical systems", Internet soft computing ,Volume 7 (1), January 2007, pp. $441-454$ 